\title{
O Conceito Energia nas Interações entre Professores e Estudantes Mediadas pelos Livros Didáticos de Biologia
}

\author{
The Energy Concept in Interactions Between Teachers and Students \\ Mediated by Biological Didactic Books
}

\author{
Sandra Maria Wirzbicki (sandra.wirzbicki@uffs.edu.br) \\ Universidade Federal da Fronteira Sul- UFFS \\ José Claudio Del Pino (delpinojc@ yahoo.com.br) \\ Universidade Federal do Rio Grande do Sul - UFRGS \\ Maria Cristina Pansera-de-Araújo (pansera95@gmail.com) \\ Universidade Regional do Noroeste do Estado do Rio Grande do Sul - UNIJUí
}

\begin{abstract}
Resumo: O Programa Nacional do Livro Didático (PNLD) é uma política pública que avalia, seleciona e distribui livros didáticos (LDs) para estudantes da educação básica brasileira. Apesar disto, os livros apresentam limitações e abordagens conceituais ou estruturais inadequadas, que repercutem no ensino e aprendizagem em sala de aula. $\mathrm{O}$ entendimento de "energia" "não é algo simples nem consensual"; por isso foram observadas descrições relativas à "energia" do metabolismo celular em oito coleções de LDs de Biologia do Ensino Médio (LDBEM) do Guia do PNLD/2012 para a $1^{\mathrm{a}}$ série. Fundamentada na Análise Textual Discursiva, procedeu-se a transcrição de excertos dos LDBEMs analisados ao conceituar "energia" e suas relações com o metabolismo. Após, entrevistas semiestruturadas foram realizadas com cinco professores de Biologia do Ensino Médio, acerca de suas concepções de "energia". Outra etapa da pesquisa foi compreender as aprendizagens dos estudantes do EM sobre o conceito "energia", por meio da análise das respostas de um questionário estruturado e de esquemas conceituais sobre o tema. A triangulação dos dados da pesquisa permite reflexões sobre a significação conceitual e a necessidade de inter-relações na área de Ciências, tanto na educação básica quanto na formação docente, relativas ao complexo conceito "energia".
\end{abstract}

Palavras-chave: Abordagens de "energia"; Descritores; Ensino e aprendizagem de Biologia.

Abstract: The National Textbook Program is a public policy that evaluates, selects and distributes textbooks to students in basic Brazilian education. However, the books still feature limitations and unsuitable conceptual or structural approaches that affect classroom teaching and learning. The understanding of "energy" cannot be seen as being neither simple nor consensual, thus the descriptions related to the cellular metabolism "energy" were observed from eight High School Biology collections from the /2012 guide for the 1st grade. Based on Discursive Textual Analysis, we proceeded to transcribe excerpts from the analyzed collections in which "energy" was conceptualized and its relations with metabolism. After that, semistructured interviews were made with five Biology teachers about their conceptions of "energy". Another research stage was to understand how learning takes place for intermediate education students regarding the "energy" concept through a questionnaire followed by the construction of conceptual schematics by the students. Research data triangulation allows for reflections upon the conceptual significance and the need for interrelations in the Natural Sciences and its 
Technologies, both in elementary education and in teacher training relative to the complex concept of "energy".

Key words: Energy approaches; Descriptors; Biology teaching and learning.

\section{INTRODUÇÃO}

Mesmo com várias possibilidades de abordar conteúdos em processos de ensino de Ciências da Natureza e suas Tecnologias (CNTs), o Livro Didático (LD) ainda constitui o principal recurso entre os poucos utilizados em salas de aula. O Programa Nacional do Livro Didático do Brasil (PNLD) tornou o LD um recurso disponível para todos os estudantes da Educação Básica brasileira. A análise contínua deste material, contudo, alerta para seus avanços e seus limites na estrutura, organização, ilustração e esquema conceitual adotado, bem como para a necessidade de compreender as mediações produzidas para as aprendizagens.

Nesta pesquisa, os estudos centraram-se nas abordagens do complexo conceito de "energia" no metabolismo energético celular dos LDs de Biologia para o EM (LDBEM) no que se refere às preocupações e consequências para a aprendizagem, em CNT particularmente, quanto à adequação dos tratamentos didáticos para a significação conceitual; isso tanto na perspectiva dos professores quanto na compreensão dos estudantes do EM.

A complexidade do ensino de "energia" pode ser remetida à visão expressa por Morin (2007) no sentido de uma busca por novas formas de conhecimento, por um “conhecimento pertinente", fundado em compreensões não segmentadas, não cerceadas aos limites de visões simplistas e fragmentadas, que levam em conta a visão de complexos sistemas de relações sistematicamente implicados.

A pretensão de compreender os processos de ensino e de aprendizagem do conceito "energia" nesta pesquisa, fundamentou-se também nas concepções que orientam as Ciências, como Marques (2002, p. 11) sugere: "a ideia ou a tese de que a base sobre a qual se assenta a necessária intercomplementaridade e interlocução das Ciências é a complexidade". 
No ensino escolar o conceito "energia" limita-se, geralmente, ao campo de cada disciplina, sem contemplar inter-relações na área, de modo que a sensação predominante expressa a "energia" da Biologia, da Química ou da Física como conceitos diferentes, sem intercomplementaridade, com a permanência de concepções simplistas entre professores e estudantes. O entendimento de que "energia" é um conceito unificador facilita os estudos no EM. As concepções de "energia" apresentadas nos LDs podem ser agrupadas conforme a área de conhecimento biológico, físico ou químico, pois

[...] na Biologia a "energia flui, na Física é capacidade de realizar trabalho e na Química é agente de transformação e de movimento". Energia é um conceito presente no ensino de Biologia, Física e Química e, quando se observam os livros didáticos desses componentes percebe-se que os conceitos estão distanciados e, portanto, existem problemas na sua contextualização (PANSERA-DE-ARAÚJO; NONENMACHER, 2009, p. 6).

É uma noção cuja compreensão abrange uma complexidade de entendimentos, nem sempre reconhecida como intrínseca ao ensino de CNT, com decorrências que podem ser remetidas a especificidades de sentidos e significados conceituais produzidos, validados e usados no âmbito de cada uma das comunidades científicas da área. Como exemplo, no EM percebe-se uma ampliação crescente de explicações sobre a biologia celular em LDBEMs, com tratamentos de conteúdos bioquímicos cuja compreensão envolve graus elevados de abstração.

Esta problemática transformou-se em objeto de investigação em contextos de planejamento e execução de práticas curriculares em CNT no EM, contrapondo-se a visões limitadas e fragmentadas da área, presentes na maioria dos LDBEMs. Partiu-se da questão de pesquisa: Que evidências de aprendizagem do conceito "energia do metabolismo celular" podem ser identificadas no ensino de Biologia no EM a partir da interação professor e aluno mediados pelo LD?

\section{LIVROS DIDÁTICOS E O CONCEITO "ENERGIA"}

Em decorrência da promulgação da LDBEN (BRASIL, 1996), movimentos de mudança no currículo, no ensino e na formação de professores passaram a ser objeto de estudos e discussões em diferentes níveis educativos e campos de saber. O desafio de concretizar um ensino com finalidade de potencializar o pleno desenvolvimento do 
educando (artigo 1º, BRASIL, 1996), contrapõe-se aos aprendizados de Ciências marcados pela tendência a uma mera repetição de respostas prontas, "com as mesmas palavras”. O que se percebe é a permanente relação dicotômica entre as práticas escolares e os discursos prescritivos de especialistas ou das políticas públicas.

Nesse cenário de mudanças nacionais propostas para a EB brasileira, têm sido discutidas abordagens de conteúdos escolares que, por não contemplarem sistemas de relação entre conhecimentos diversificados, podem comprometer os processos de significação conceitual. As preocupações voltam-se para fatores que limitam os aprendizados pela carência de inter-relações entre conceitos, em especial nos LDs, posto que, mesmo com várias possibilidades de aproximação dos conteúdos, em processos de ensino de CNT o LD, na maioria das vezes, constitui um dos poucos recursos pedagógicos usados em salas de aula. Mesmo que, pesquisadores e acadêmicos venham se dedicando há pelo menos duas décadas a investigar a qualidade das coleções didáticas, denunciando suas deficiências e apontando soluções para a melhoria de sua qualidade, suas vozes não são ouvidas nem pelas editoras e autores de LDs, tampouco pelos órgãos gestores das políticas públicas educacionais (FRACALANZA, MEGID NETO, 2006).

Por isso, é fundamental empreender esforços no sentido de uma compreensão mais ampla das repercussões, no ensino, de formas diferenciadas de tratamento dos conteúdos por parte dos LDs. O que se percebe é que, nessa área, abordagens convencionais refletem a visão de uma sequência linear e fragmentada de conteúdos, o que tende a dificultar o desenvolvimento de conceitos escolares que contemplem interrelações de conhecimentos diversificados. Infelizmente, na formação inicial dos professores, prevalece o modo fragmentado de abordar conceitos no âmbito de cada disciplina; e desconstruir isso depois é bem mais complicado. Por isso, aposta-se em uma formação que integra realmente a escola e a universidade.

No âmbito desta pesquisa voltou-se o olhar às abordagens de "energia" em LDBEMs, relativas à biologia celular, no que se refere a preocupações e possíveis decorrências das abordagens conceituais no EM, em CNT particularmente, quanto à adequação dos tratamentos didáticos para a significação conceitual. Nas Ciências, o 
entendimento do que é a "energia" não pode ser visto como uma concepção simples nem consensual, sendo importante considerar as amplitudes dos significados, a exemplo da explicação expressa por Angotti (1991) e corroborada por Auth (2000) de que a "energia" é:

um "sutil camaleão" do conhecimento científico. Transforma-se espacial e temporalmente, na dinâmica mutável dos objetos, fenômenos e sistemas, conserva-se na totalização das distintas formas e degrada-se porque uma de suas formas - o calor - é menos elástico e reversível do que outras (AUTH, 2000, p. 7).

Nem mesmo dentro da área as abordagens de conteúdos e conceitos transdisciplinares, como é o caso de "energia", contemplam inter-relações de conhecimentos entre disciplinas, o que situa e justifica a importância de estudos em busca de conhecimentos e ações que contribuam para a perspectiva da articulação dos conceitos/conteúdos na área, bem como contemplam conteúdos para além dos conceituais, por exemplo, atitudinais, procedimentais, éticos, morais, políticos, sociais, culturais, dentre outros, acerca da "energia".

É uma noção cuja compreensão abrange uma complexidade de entendimentos, nem sempre reconhecida como inerente ao ensino de CNT, com decorrências que podem ser remetidas a especificidades de sentido e significados conceituais produzidos, validados e usados no âmbito de cada uma das comunidades científicas da área. Assim, uma diversidade de entendimentos relativos ao conceito "energia", complexifica a compreensão conceitual em sentido mais amplo.

Diante das expressões simplistas e equivocadas presentes em vários LDBEMs, resultando em compreensões incoerentes com formas científicas adequadas de explicação, cabe considerar que não podemos mais voltar a definições arcaicas dos conceitos, uma vez que estudos sobre a natureza do calor sempre estiveram em foco para os químicos e físicos dos séculos 18 e 19.

Assim como a importância do conceito de "energia" está no fato de que a conservação da "energia" é uma lei que governa todos os fenômenos de natureza, e que carece em contexto escolar ou universitário, de avanços nas compreensões e explicações que levem em conta novos conhecimentos, versatilidade e complexidade do conceito. Discute-se o papel da escola e do professor na promoção do desenvolvimento humano, 
concebendo os estudantes como sujeitos com vivências, experiências, com potencialidades diversas para pensar e expressar conhecimentos acerca de objetos complexos cuja compreensão requer inter-relações de significados conceituais, como é o caso dos estudos sobre a "energia".

\section{O PERCURSO METODOLÓGICO}

A metodologia proposta neste trabalho é de natureza essencialmente qualitativa, a partir de um estudo de caso que, segundo Lüdke e André (1986), se caracteriza pela coleta de dados em um processo que envolve um campo de trabalho específico, sempre bem-delimitado e de contornos claramente definidos. Este estudo de caso foi realizado em quatro etapas: I) análise documental de abordagens de "energia" em LDBEMs, para a $1^{\text {a }}$ série do EM (volumes que abordam a "energética celular"), aprovados e sugeridos pelo Guia de LD PNLD 2012 (BRASIL, 2011); II) entrevista semiestruturada com Professores do Ensino Médio de Biologia (PEMBs); III) aplicação de questionário elaborado com a estrutura da Escala de Likert aos estudantes de escolas do Ensino Médio; e IV) elaboração de esquema-síntese pelos estudantes de escolas do EM (EEEM), alunos dos PEMBs envolvidos na pesquisa, que já estudaram conteúdos da "energética celular".

A partir da Análise Textual Discursiva de Moraes e Galiazzi (2007), procedeu-se a transcrição e unitarização de excertos dos LDBEMs analisados, conceituando "energia" e suas relações com o metabolismo. Foi possível reconhecer um conjunto de descritores identificados por palavras, que permitiram organizar cinco categorias de análise. Os mesmos descritores orientaram o olhar para as falas transcritas das entrevistas dos PEMBs e análise dos esquemas dos EEEMs.

\section{4. "ENERGIA" NOS LDBEMS, NAS FALAS DOS PEMBS E EEEMS}

O entendimento de "energia" não é algo simples nem consensual, por isso as descrições relativas ao "metabolismo energético celular" foram observadas nos LDBEMs do Guia do PNLD/2012 (BRASIL, 2011) para a 1ª́rie. Posteriormente à identificação dos descritores das imagens, das falas dos professores, das respostas aos questionários e dos esquemas construídos pelos estudantes, destacaram-se as Unidades de Análise: 1) "energia" nos seres vivos, respiração e fotossíntese; 2) "energia" nas 
substâncias e ligações químicas - ATP/ADP; e 3) "energia" nas representações, identificadas em todos os dados. A partir das Unidades de Análise foram reconhecidos problemas na organização conceitual e propuseram-se cinco categorias de análise: I) organização conceitual de "energia" nos seres vivos e materiais e substâncias com diferentes palavras; II) complexidade da expressão conceitual de "energia" no metabolismo celular; III) analogias e imagens na explicação das reações moleculares da "energia" do metabolismo celular; IV) aspectos do conceito "energia" relacionados ao cotidiano, e V) a interdisciplinaridade no ensino e aprendizagem de "energia" do metabolismo celular. Tendo em vista o destaque para as três unidades de análise, também, serão problematizados os dados analisados nas categorias I, II e III.

\subsection{Categorias I, II e III: Organização conceitual de "energia", complexidade e analogias do metabolismo celular}

É preocupante a constatação de que, por exemplo, nos LDBEMs o desenvolvimento do conceito "energia" fundamenta-se essencialmente nos aspectos bioquímicos, cuja compreensão pelos estudantes envolve graus elevados de abstração.

Excerto 1 - Esse processo (degradação e perda de energia por meio de calor) é possível graças a um mecanismo conhecido como acoplamento de reações, ${ }^{1}$ em que algumas substâncias armazenam temporariamente a energia que será posteriormente transferida, de modo que a perda sob a forma de calor seja a menor possível. Uma dessas substâncias é o trifosfato de adenosina ou adenosina trifosfato (ATP) (LDBEM 8, p. 124).

As abordagens sobre o metabolismo energético celular nos LDBEMs da $1^{\text {a }}$ série do EM incluem estudos e entendimentos sobre as vias metabólicas e suas relações com processos de produção e consumo de ATP na manutenção dos processos vitais. Isto acaba provocando uma quebra na compreensão do conceito, fazendo com que o aluno não relacione as suas vivências com os novos aprendizados.

Já na Figura 1 - Reações Acopladas, a imagem apresenta uma simplificação de noções complexas, que requerem compreensões adequadas sobre as interconversões de "energia" envolvidas no complexo sistema de transformações que propicia a "energia" da vida (ATP). O LD em análise simplifica tanto que se refere ao alimento como "molécula". O uso do termo "liberação" compromete a compreensão conceitual pelo

\footnotetext{
${ }^{1}$ Legenda dos grifos nos excertos dos LDBEMs e PEMBs: Itálico: energia; Negrito: para os descritores; Sublinhado: para grifos do LDBEM.
} 
risco de uma visão mecanicista de "energia". Pode-se afirmar que em abordagens como as da Figura 1, conceitos como os de reação "exergônica" e "endergônica" são tratados de forma simplificada, sem considerar a complexidade dos processos de transformação e conservação de "energia”, envolvidos nos processos em estudo. A interpretação requer o entendimento conceitual das transformações como sistemas complexos, como é o caso das interconversões energéticas associadas à compreensão conceitual da respiração celular, que demanda movimentos dialéticos de "ir e vir" entre os níveis macro e microscópicos de conhecimento.

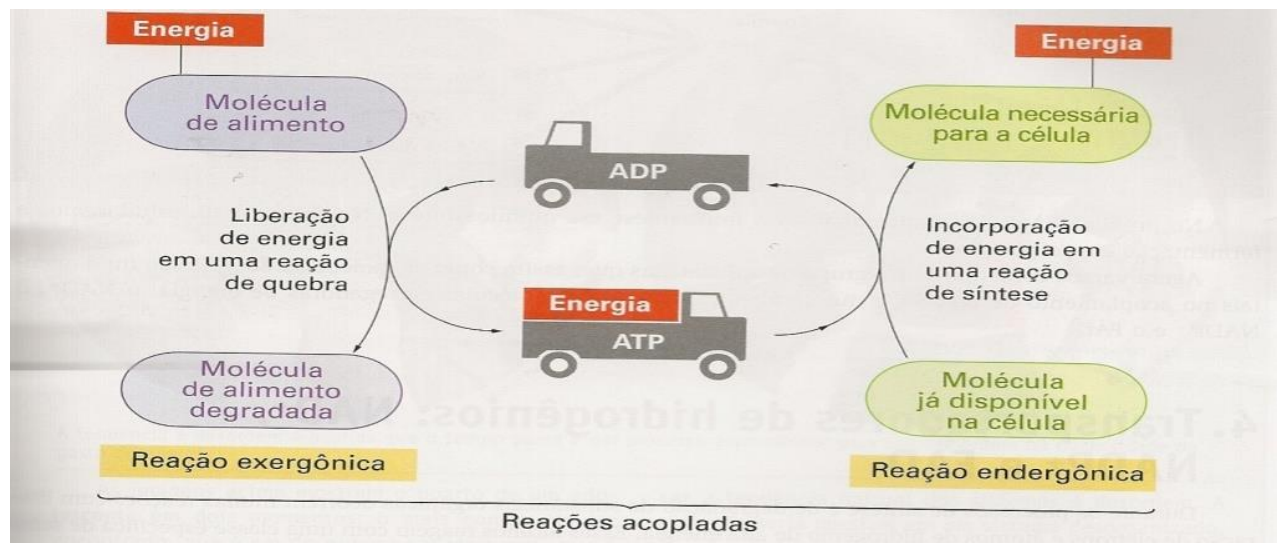

Figura 1 - Reações Acopladas Fonte: LDBEM 8, p. 323.

A imagem de um "mero movimento de caminhõezinhos" em um ir e vir, com ou sem a "carga" pode criar um obstáculo ao desenvolvimento dos conhecimentos escolares sobre as transformações em nível molecular, coerentes com a forma científica de explicar, tendo em vista que podem promover compreensões de "energia" como matéria que pode ser carregada. Aspecto positivo dessa analogia é de que essa "energia" ADP e ATP não fica armazenada e ela é transformada imediatamente em outra forma. Vale ressaltar que as analogias correm risco sempre, e, nos termos bachelardianos (BACHELARD, 1996), é necessária uma vigilância epistemológica ante a imagens como essa, que acomodam o pensamento, obstacularizando os entendimentos conceituais necessários de serem mediados na escola para o desenvolvimento humano. Pode-se concluir que cabe aos professores a forma de tratar o assunto ao utilizar-se destas figuras/metáforas para que a compreensão dos objetos complexos potencialize o estabelecimento de relações entre as noções de reação "exergônica" e "endergônica". 
Essa complexidade faz parte das explicações fornecidas pelos PEMBs a partir dos LDBEMs, em sala de aula, pois sua complexidade impulsiona a busca por relações com situações vivenciais dos estudantes para auxiliar na significação conceitual. Isso é observado na resposta de PEMB1 sobre o que considera mais significativo na compreensão/significado do conceito "energia"?

PEMB1 (24-26): [...] para mim é importante o aluno compreender de onde vem essa energia, não só no corpo deles, (...) como em células animais e células vegetais, que vai funcionar um pouco diferente e como a célula vai transformar isso para mandar para o corpo, para o organismo funcionar. [...] Quando nós vimos as organelas citoplasmáticas, eu trabalhei a mitocôndria com eles, então quando a gente entrou em membrana, a gente falou dos transportes, transporte ativo gasto de energia, mas que energia é essa? É o ATP, mas o que é ATP? De onde que ele vem?

No depoimento, é possível perceber compreensões de "energia" decorrentes de uma formação sem muitas interlocuções dentro da área de CNT, restritas ao campo disciplinar da Biologia, com concepções simplificadas, mais relacionadas a situações do cotidiano sem levar em conta os níveis moleculares, inerentes às explicações do metabolismo celular, apresentadas nos LDBEMs utilizados. Essa resposta reitera a discussão defendida ao longo da Tese quanto à necessidade de níveis elevados de abstração na compreensão de aspectos moleculares envolvidos na obtenção/transformação de "energia", e como isso será efetivado no ensino de CNT.

Contudo, índices elevados de utilização dos LDBEMs são evidentes nos esquemas dos EEEM2 da escola pública militar. Os esquemas remetem a altos teores de memorização em relação ao que é proposto pelos LDBEMs. Apresentam-se, contudo, também equívocos conceituais, a exemplo da definição da "realização da glicólise por meio de organismos anaeróbios”. 


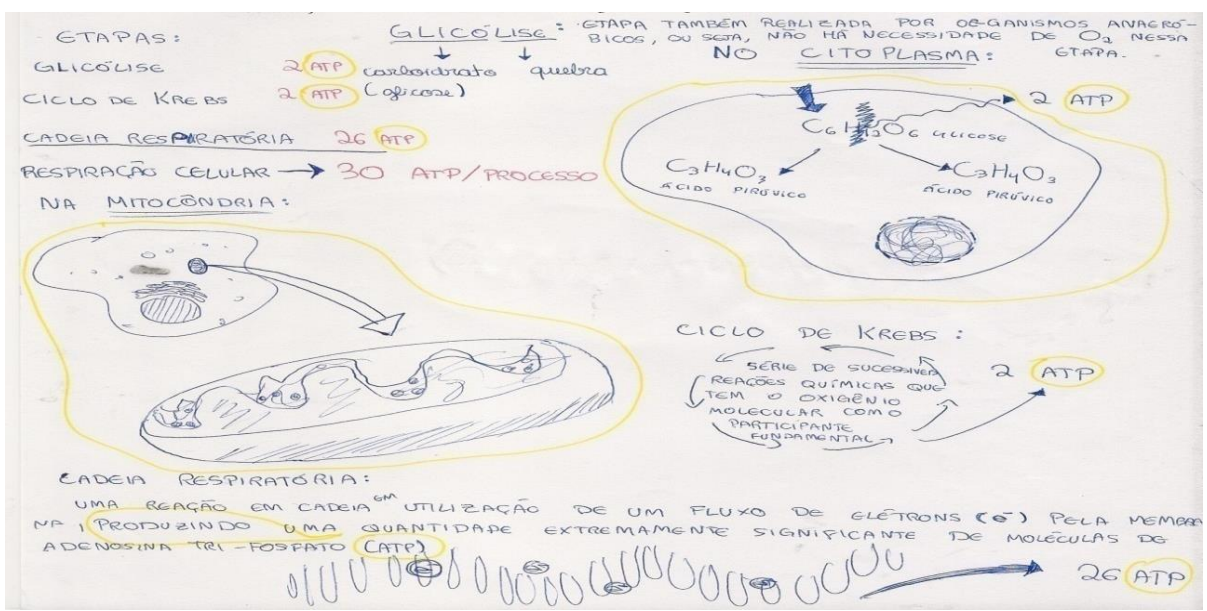

Figura 2: E1 - EEM2

O esquema de E1 da EEEM2, e vários deste grupo de estudantes, são exemplos disso, uma vez que representam praticamente todos os processos envolvidos na respiração celular, inclusive com a apresentação de um saldo de ATP por E1. Será que representou suas compreensões ou apenas reproduziu o que memorizou e ainda é avaliado no Enem ou em vestibulares? Surgem indagações sobre a aprendizagem desenvolvida nas escolas quando o organizador do currículo é o LDBEM escolhido, bem como os exames de avaliação nacional ou para ingresso no Ensino Superior, como o Exame Nacional do Ensino Médio e o vestibular: Será que os esquemas construídos ultrapassam o nível memorístico, promovendo a evolução da compreensão conceitual dos estudantes? Mesmo que PEMB2 se refira a aprendizagens significativas, que auxiliem os estudantes a minimamente melhorar sua vida, deixa claro, no turno 22 da entrevista, que o ensino em EEM2 também “objetiva gabaritar as questões de Biologia”.

Sobre as analogias, cabe inferir que elas foram identificadas em cinco dos nove LDBEMs investigados. Se o professor não tiver uma leitura crítica ou interferir nestas abordagens, elas podem prejudicar a aprendizagem de conceitos, confundindo o estudante. O Excerto 3, extraído de LDBEM1, apresenta uma explicação correta do ATP.

Excerto 3 - O estoque de ATP em uma única célula é da ordem de um 1 bilhão de moléculas, que são usadas e repostas a cada 2 ou 3 minutos, interruptamente. Por essa razão, alguns pesquisadores comparam o ATP a uma "moeda energética" que circula dentro da célula e "custeia" os gastos metabólicos (LDBEM 1, p. 197). 
O ATP, contudo, é comparado a uma "moeda energética". A aprendizagem é dificultada ainda mais pelo uso de metáforas e analogias, com o intuito de efetuar a transposição didática do conhecimento científico do estudante (LOPES, 2007) em detrimento do conteúdo e da conceitualização que tanto se almeja. Conceitos básicos de Biologia, Química e Física, abstratos por natureza, necessitam ser usados com mobilização da capacidade de pensar conceitualmente sobre eles, entendendo-os de forma dinamicamente inter-relacionada. Tratam-se de conceitos cujos significados assumem vieses diferenciados no âmbito cultural próprio a cada uma das disciplinas da área de CNT, as quais precisam colaborar na construção conceitual dos alunos.

\section{CONSIDERAÇÕES FINAIS}

A pesquisa possibilitou conhecer as abordagens de "energia" propostas nos LDBEMs, tanto nos textos quanto nas imagens, compreendendo a complexidade que dificulta o ensino e a aprendizagem deste conceito. $\mathrm{O}$ olhar aos descritores permitiu identificar as limitações na conceitualização da "energia" nos seres vivos, o que pode complicar a aprendizagem e a constituição do conhecimento científico pelos sujeitos, uma vez que ainda impõem uma visão linear e descontextualizada. Ao reconhecer estes descritores, a reflexão sobre a organização do ensino propicia produções autorais de material didático.

As observações apontam para a necessidade de entender os limites de abordagens conceituais de "energia" em LDBEMs utilizados no EM. Sem desconsiderar a importância deles no contexto escolar, sugere-se que haja um olhar mais crítico sobre as formas como conteúdos/conceitos estruturantes do conhecimento na ciência são abordados, na perspectiva de preservar a coerência com o conhecimento científico. Ao reconhecer o conjunto de descritores, abre-se uma perspectiva de reflexão sobre a organização do currículo escolar ainda alinhado ao proposto pelos LDs. Por isso, é fundamental a tomada de consciência sobre os limites das abordagens do LD bem como o seu papel nos estudos. Por outro lado, é importante valorizar as inter-relações necessárias à compreensão conceitual enquanto sistemas de relações a serem considerados no ensino de temas complexos, como é o caso da "energia". 
Estabelecer diálogos com as concepções dos PEMBs sobre "energia" foi uma forma de corroborar a análise realizada nos LDBEMs, pois os mesmos expressam as carências provenientes da própria formação e das limitações no espaço da escola quanto às dificuldades do desenvolvimento de atividades interdisciplinares, bem como perceber o esforço dos professores, em proporcionar um ensino contextual, contemplando as experiências dos estudantes.

Nessa perspectiva, espera-se que os cursos de formação de professores, inicial ou continuado, abram espaços para discussões sobre fatores que limitam as práticas docentes, cerceadas ao seguimento de um LD ou a concepções ultrapassadas, sem estabelecimento de relações dentro e fora da área específica. A compreensão de um professor de CNT é de um sujeito em permanente processo de formação, sistematicamente aberto para novos conhecimentos e reflexões, incluindo os colegas que atuam em outras disciplinas, bem como os dos pesquisadores e dos estudantes.

Cabe referendar a visão do professor de CNT como um sujeito em permanente processo de formação, aberto para novos conhecimentos e reflexões, valorizando conhecimentos diversificados, incluindo colegas que atuam em outras disciplinas, bem como os pesquisadores e estudantes; um sujeito capaz de reconstruir significados de conceitos e de esquemas conceituais, promovendo, assim, aprendizados associados ao desenvolvimento humano/social, de forma mais rica e plena.

\section{REFERENCIAS}

ANGOTTI, J. A. P. Fragmentos e totalidades no conhecimento científico e no ensino de ciências. 1991. Tese (Doutorado) - Universidade de São Paulo, USP, São Paulo, 1991.

AUTH, M. A. Conceitos unificadores e o ensino de ciências. In: Espaços da Escola, Ijuí: Ed. Unijuí, RS, v. 38, p. 63-80, 2000.

BACHELARD, G. A formação do espírito científico: contribuição para uma psicanálise do conhecimento. Tradução Estela dos Santos Abreu. Rio de Janeiro: Contraponto, 1996.

BRASIL. Ministério da Educação. Secretaria de Educação Média e Tecnológica. Lei de Diretrizes e Bases da Educação Nacional. Brasília: Ministério da Educação, 1996. 
- Ministério da Educação - MEC; Secretaria de Educação Básica. Guia Nacional dos livros didático - PNLD 2012: Biologia. Brasília: Ministério da Educação, 2011. 76 p.

FRACALANZA, H. O ensino de ciências no Brasil. In: FRACALANZA, Hilário; MEGID NETO, Jorge (Orgs.). O livro didático de ciências no Brasil. Campinas: Komedi, 2006.

LOPES, A. C. Currículo e epistemologia. Ijuí: Ed. Unijuí, 2007.

LOPES, Sônia. Biologia. Volume 1. Org. Sônia Lopes e Sergio Rosso. 2. ed. São Paulo: Saraiva, 2010.

LÜDKE, M.; ANDRÉ, M. E. D. A. Pesquisa em educação: abordagens qualitativas. São Paulo: EPU, 1986.

MARQUES, M. O. Educação nas ciências: interlocuções e complementaridade. Ijuí: Ed. Unijuí, 2002. 160p.

MARTHO, G. R.; AMABIS, J. M. Biologia: Biologia das células. São Paulo: Editora Moderna, 2010. Volume 1. 2007.

MORAES, R.; GALIAZZI, M. C. Análise textual discursiva. Ijuí: Ed. Unijuí,

MORIN, E. Os sete saberes necessários à educação do futuro. 12. ed. São Paulo: Cortez; Brasília, DF: Unesco, 2007.

PANSERA-DE-ARAÚJO M. C.; NONENMACHER, S. Energia: um conceito presente nos livros didáticos de física, biologia e química do Ensino Médio. In: Poiésis, Tubarão, v. 2, n. 1, p. 1-13, jan./jun. 2009. 